\title{
CAUCHY PROBLEM FOR THE ZAKHAROV SYSTEM ARISING FROM HOT PLASMA WITH LOW REGULARITY DATA*
}

\author{
LIJIA HAN ${ }^{\dagger}$, JINGJUN ZHANG ${ }^{\ddagger}$, ZAIHUI GAN ${ }^{\S}$, AND BOLING GUO
}

\begin{abstract}
We consider the Zakharov system arising from hot plasma, which can be seen as the classical Zakharov system coupled to a given dissipative equation. We prove local in time wellposedness for this kind of Zakharov system with large initial data $\left(\mathcal{E}_{0}, n_{0}, n_{1}, \mathcal{B}_{0}\right)$ in $H^{\frac{1}{2}+\epsilon} \times L^{2} \times$ $H^{-1} \times H^{-1+\epsilon}$, where $\epsilon>0$ is any sufficient small number. This improves the known results obtained by C. Laurey in 1995 and C. Kenig and W. Wang in 1998.
\end{abstract}

Key words. Zakharov system, Bourgain space, local well-posedness.

AMS subject classifications. 35Q55, 35Q53, 35Q35, 76B03.

\section{Introduction}

In this paper, we study the Cauchy problem for the Zakharov system arising from hot plasma:

$$
\left\{\begin{array}{l}
\mathrm{i} \mathcal{E}_{t}+\nabla(\nabla \cdot \mathcal{E})-\alpha \nabla \times(\nabla \times \mathcal{E})-n \mathcal{E}+\mathrm{i} \mathcal{E} \times \mathcal{B}=0 \\
n_{t t}-\Delta n=\Delta|\mathcal{E}|^{2} \\
\Delta \mathcal{B}-\mathrm{i} \beta \nabla \times(\nabla \times(\mathcal{E} \times \overline{\mathcal{E}}))-\gamma \frac{\partial}{\partial t} \int_{\mathbb{R}^{3}} \frac{\mathcal{B}(t, y)}{|x-y|^{2}} d y=0
\end{array}\right.
$$

with initial data

$$
\mathcal{E}(0, x)=\mathcal{E}_{0}(x),\left(n(0, x), n_{t}(0, x)\right)=\left(n_{0}(x), n_{1}(x)\right), \mathcal{B}(0, x)=\mathcal{B}_{0}(x) .
$$

The function $\mathcal{E}: \mathbb{R}^{3} \times[0,+\infty) \rightarrow \mathbb{C}^{3}$ is the slowly varying amplitude of the highfrequency electric field, the function $n: \mathbb{R}^{3} \times[0,+\infty) \rightarrow \mathbb{R}$ denotes the fluctuation of the ion-density from its equilibrium, and the function $\mathcal{B}: \mathbb{R}^{3} \times[0,+\infty) \rightarrow \mathbb{R}^{3}$ is the self-generated magnetic field. $\overline{\mathcal{E}}$ denotes the conjugate complex of $\mathcal{E}$, and the notation $\times$ means the cross product for $\mathbb{R}^{3}$ or $\mathbb{C}^{3}$ valued vectors. The constants $\alpha, \beta$, and $\gamma$ satisfy $\alpha \geq 1, \beta, \gamma>0$.

System (1.1) describes the spontaneous generation of a magnetic field in hot plasma, and one can refer to [15] for its physical background. There are also some types of magnetic Zakharov equations in cold plasmas; one can refer to [10, 12, 13] for their physical background and mathematical results. Omitting the effect of the magnetic field $\mathcal{B}$ and taking $\alpha=1$, we can arrive at the classical Zakharov system derived by V. E. Zakharov:

$$
\left\{\begin{array}{l}
\mathrm{i} \mathcal{E}_{t}+\Delta \mathcal{E}-n \mathcal{E}=0 \\
\lambda^{-2} n_{t t}-\Delta n=\Delta|\mathcal{E}|^{2}
\end{array}\right.
$$

${ }^{*}$ Received: November 11, 2011; accepted: June 22, 2012. Communicated by Lenya Ryzhik.

${ }^{\dagger}$ Department of Mathematics and Physics, North China Electric Power University, Beijing, 102206, P.R. China (hljmath@gmail.com).

$\ddagger$ College of Mathematics, Physics and Information Engineering, Jiaxing University, Zhejiang, 314001, P.R. China (zjj_math@yahoo.com.cn).

$\S$ College of Mathematics and Software Science, Sichuan Normal University, Chengdu, 610068, P.R. China (ganzaihui2008cn@yahoo.com.cn).

IInstitute of Applied Physics and Computational Mathematics, P.O. Box 8009, Beijing, 100088, P.R. China (gbl@iapcm.ac.cn). 
where $\lambda$ denotes the ion acoustic speed, and usually we can put $\lambda=1$. The Zakharov system describes the propagation of Langmuir waves in plasmas; see [26]. When $\lambda \rightarrow \infty$, we can formally obtain the cubic Schrödinger equation

$$
\mathrm{i} \mathcal{E}_{t}+\Delta \mathcal{E}+|\mathcal{E}|^{2} \mathcal{E}=0
$$

System (1.3) has been studied by many authors, and there are a lot of results about the global existence of weak solutions, smooth solutions, and local wellposedness for this system; cf. $[1,5,6,18,19,9,14,23,24,25]$ and the references therein. For the convergence results from the solutions of system (1.3) to those of the limit equation (1.4), we refer to [2, 20, 21, 25].

In the case $d=3$, the local well-posedness results were obtained by J. Ginibre, Y. Tsutsumi, and G. Velo [14]. They proved the local well-posedness for the Cauchy problem of system (1.3) with initial data $\left(\mathcal{E}_{0}, n_{0}\right) \in H^{\frac{1}{2}} \times L^{2}$. This well-posedness result is reflected by the limit equation (1.4). From the scaling invariant point of view, it is well-known that $H^{\frac{1}{2}}$ is the critical space for the cubic Schrödinger equation (1.4) in dimension three. Recently, I. Bejenaru and S. Herr [7] proved the local well-posedness result of (1.3) in 3D for initial data $\left(\mathcal{E}_{0}, n_{0}\right) \in H^{0+\epsilon} \times H^{-\frac{1}{2}+\epsilon}(\epsilon>0$ can be arbitrarily small).

For system (1.1), C. Laurey [17] proved the existence of local smooth solutions with smooth initial data $\left(\mathcal{E}_{0}, n_{0}, n_{1}, \mathcal{B}_{0}\right) \in H^{s+1}\left(\mathbb{R}^{3}\right) \times H^{s}\left(\mathbb{R}^{3}\right) \times\left(H^{s-1}\left(\mathbb{R}^{3}\right) \cap\right.$ $\left.\dot{H}^{-1}\left(\mathbb{R}^{3}\right)\right) \times H^{s+1}\left(\mathbb{R}^{3}\right)$ with $s>3 / 2$. These results were obtained by using energy methods, and the condition $n_{1} \in \dot{H}^{-1}$ is necessary. In [16], C. Kenig and W. Wang applied smoothing effect estimates and a standard iteration method, and proved the existence of a local smooth solution without the hypothesis $\dot{H}^{-1}$, for small data $\left(\mathcal{E}_{0}, n_{0}, n_{1}, \mathcal{B}_{0}\right) \in\left(H^{s} \cap\left(\cap_{m+\beta \leq s_{0}+16} H^{\beta}\left(|x|^{m} d x\right)\right)\right) \times H^{s-1 / 2} \times H^{s-3 / 2} \times H^{s}$ with $s>30$. In this paper, our purpose is to prove the local well-posedness results for system (1.1) with low regularity and large initial data $\left(\mathcal{E}_{0}, n_{0}, n_{1}, \mathcal{B}_{0}\right) \in$ $H^{1 / 2+\epsilon} \times L^{2} \times H^{-1} \times H^{-1+\epsilon}$, for any $\epsilon>0$ sufficiently small.

Our starting point is the Bourgain space method, which was introduced by Bourgain in $[3,4]$ to study the Cauchy problem for nonlinear dispersive evolution equations. This method later successfully solved many problems for many dispersive equations, including the nonlinear Schrödinger equation, the Korteweg-de Vries equation [8], the Zakharov equation [14], the Benjamin-Ono equation, and so on. In this paper, we will apply the Bourgain space method to solve a dissipative equation, which is coupled with two dispersive equations; see the third equation in (2.2). It seems that the argument with the Bourgain spaces was never applied to a dissipative equation. The method can also be used to treat the case when space dimension is not 3 . First we state our main results in this paper.

Theorem 1.1. Assume the initial data $\left(\mathcal{E}_{0}, n_{0}, n_{1}, \mathcal{B}_{0}\right)$ belongs to

$$
H^{k}\left(\mathbb{R}^{3}\right) \times H^{l}\left(\mathbb{R}^{3}\right) \times H^{l-1}\left(\mathbb{R}^{3}\right) \times H^{k-\frac{3}{2}}\left(\mathbb{R}^{3}\right),
$$

with

$$
l \leq k \leq l+1, \frac{1}{2}(l+1) \leq k, l \geq 0, k>\frac{1}{2} .
$$

Then there exists $T>0$ (depending on the norm of the initial data), such that the Cauchy problem (1.1)-(1.2) has a unique solution $\left(\mathcal{E}, n, n_{t}, \mathcal{B}\right)$ belonging to 
$C\left([0, T] ; H^{k} \times H^{l} \times H^{l-1} \times H^{k-3 / 2}\right)$, and meanwhile

$$
\mathcal{E} \in X_{S}^{k, \frac{1}{2}}(T), n \in X_{W}^{l, \frac{1}{2}}(T), n_{t} \in X_{W}^{l-1, \frac{1}{2}}(T), \mathcal{B} \in X_{H}^{k-\frac{3}{2}, \frac{1}{2}}(T),
$$

where $X_{S}^{k, \frac{1}{2}}(T), X_{W}^{l, \frac{1}{2}}(T)$, and $X_{H}^{k-\frac{3}{2}, \frac{1}{2}}(T)$ are defined in (2.7), (2.9), and (2.11) in Section 2.2. Moreover, the solution map $\left(\mathcal{E}_{0}, n_{0}, n_{1}, \mathcal{B}_{0}\right) \rightarrow\left(\mathcal{E}, n, n_{t}, \mathcal{B}\right)$ is locally Lipschitz continuous.

\section{Notations}

Throughout the paper, $C$ will denote a universal positive constant which can be different at each appearance. For $x, y>0, x \lesssim y$ means that $x \leq c y$, and $x \ll y$ denotes $c x<y$ for sufficiently large $c$, and $x \sim y$ stands for $x \lesssim y$ and $y \lesssim x$.

For $f \in \mathscr{S}^{\prime}$, we let $\tilde{f}(\tau, \xi)$ or $\mathscr{F}_{t, x} f(t, x)$ denote the Fourier transform of $f$ in both the $t$ and $x$ variables, namely

$$
\tilde{f}(\tau, \xi)=\mathscr{F}_{t, x}(f)=\int_{\mathbb{R}^{d} \times \mathbb{R}} e^{-\mathrm{i} x \cdot \xi} e^{-\mathrm{i} t \cdot \tau} f(x, t) d x d t .
$$

And we denote by $\hat{u}(t, \xi)$ or $\mathscr{F}_{x} u(t, x)$ the Fourier transform in only the $x$ variable.

For any measurable function $\varphi: \mathbb{R}^{3} \rightarrow \mathbb{C}$, we define the Fourier multiplier by $\varphi(\nabla / \mathrm{i})=\mathscr{F}_{x}^{-1} \varphi(\xi) \mathscr{F}_{x}$, i.e., $\mathscr{F}_{x}(\varphi(\nabla / \mathrm{i}) f)=\varphi(\xi) \hat{f}$.

The notation $\chi_{\mathbb{R}^{+}}(t)$ is the usual characteristic function, that is, $\chi_{\mathbb{R}^{+}}(t) \equiv 1$ if $t \geq 0$, otherwise $\chi_{\mathbb{R}^{+}}(t) \equiv 0$. We also define $\chi_{P}$ for statements $P$ to be 1 if $P$ is true and 0 otherwise.

\section{Preliminaries}

2.1. Reduction of the original system. For the first equation in system (1.1), taking Fourier transform of the linear part i $\mathcal{E}_{t}+\nabla(\nabla \cdot \mathcal{E})-\alpha \nabla \times(\nabla \times \mathcal{E})=0$ yields that

$$
\hat{\mathcal{E}}_{t}=-\mathrm{i}(\xi(\xi \cdot \hat{\mathcal{E}})-\alpha \xi \times(\xi \times \hat{\mathcal{E}}))=-\mathrm{i}\left(\alpha|\xi|^{2} \hat{\mathcal{E}}+(1-\alpha) \xi(\xi \cdot \hat{\mathcal{E}})\right)=:-\mathrm{i} P(\xi) \hat{\mathcal{E}},
$$

where $P(\xi)$ is a real symmetric $3 \times 3$ matrix

$$
P(\xi)=\alpha|\xi|^{2} I_{3 \times 3}+(1-\alpha)\left(\begin{array}{ccc}
\xi_{1}^{2} & \xi_{1} \xi_{2} & \xi_{1} \xi_{3} \\
\xi_{2} \xi_{1} & \xi_{2}^{2} & \xi_{2} \xi_{3} \\
\xi_{3} \xi_{1} & \xi_{3} \xi_{2} & \xi_{3}^{2}
\end{array}\right) .
$$

Note that for all $e \in \mathbb{C}^{3}$ we have

$$
|\xi|^{2}|e|^{2} \leq e^{\prime} P(\xi) \bar{e}=\alpha|\xi|^{2}|e|^{2}+(1-\alpha)|\xi \cdot e|^{2} \leq \alpha|\xi|^{2}|e|^{2} .
$$

Indeed, it is not hard to see that the eigenvalues of $P(\xi)$ are $\lambda_{1}(\xi)=|\xi|^{2}$ and $\lambda_{2}(\xi)=$ $\lambda_{3}(\xi)=\alpha|\xi|^{2}$. This fact implies that the first equation of (1.1) can be reduced to the case $\alpha=1$; see the discussion in the next subsection.

Next, we reduce the second equation about $n$ of the original system (1.1) into a first order system by setting $N=n+\mathrm{i}\langle\nabla\rangle^{-1} n_{t}$. Then we can obtain $n$ and $n_{t}$ by the relations $n=\operatorname{Re} N$ and $n_{t}=\operatorname{Im}(\langle\nabla\rangle N)$, respectively.

For the last equation about $\mathcal{B}$, from the Riesz potential we can write

$$
\int_{\mathbb{R}^{3}} \frac{\mathcal{B}(t, y)}{|x-y|^{2}} d y=c_{0}(-\Delta)^{-\frac{1}{2}} \mathcal{B}, \quad \text { for some } \quad c_{0}>0
$$


where

$$
(-\Delta)^{\frac{s}{2}}:=\mathscr{F}^{-1}|\xi|^{s} \mathscr{F}, \quad s \in \mathbb{R} .
$$

Hence, the original system (1.1)-(1.2) is reduced to

$$
\left\{\begin{array}{l}
\mathrm{i} \mathcal{E}_{t}+\Delta \mathcal{E}=\frac{1}{2}(N+\bar{N}) \mathcal{E}-\mathrm{i} \mathcal{E} \times \mathcal{B} \\
\mathrm{i} N_{t}-\langle\nabla\rangle N=-\langle\nabla\rangle^{-1} \Delta|\mathcal{E}|^{2}-\frac{1}{2}\langle\nabla\rangle^{-1}(N+\bar{N}) \\
\mathcal{B}_{t}+(-\Delta)^{\frac{3}{2}} \mathcal{B}=-\mathrm{i}(-\Delta)^{\frac{1}{2}} \nabla \times(\nabla \times(\mathcal{E} \times \overline{\mathcal{E}}))
\end{array}\right.
$$

with initial data

$$
\mathcal{E}(0, x)=\mathcal{E}_{0}(x), N(0, x)=n_{0}(x)+\mathrm{i}\langle\nabla\rangle^{-1} n_{1}(x), \mathcal{B}(0, x)=\mathcal{B}_{0}(x) .
$$

For notational simplicity, we have set $c_{0} \gamma=1$ and $\beta=1$ in the equation for $\mathcal{B}$. In the remaining part of this paper, we are devoted to studying the local well-posedness of system (2.2).

2.2. Introduction to resolution space. Consider the general dispersive equation

$$
\mathrm{i} u_{t}-\phi(\nabla / \mathrm{i}) u=f(u), u(0)=u_{0},
$$

where $\phi$ is a real function (or real symmetric matrix valued function). To solve (2.4), Bourgain introduced a space $X_{\tau=\phi(\xi)}^{s, b}$ defined by the completion of the Schwarz function space $\mathcal{S}\left(\mathbb{R}^{1+d}\right)$ with respect to the norm

$$
\|u\|_{X_{\tau=\phi(\xi)}^{s, b}}:=\|S(-t) u\|_{H_{t}^{b} H_{x}^{s}}=\left\|\langle\xi\rangle^{s}\langle\tau+\phi(\xi)\rangle^{b} \tilde{u}(\tau, \xi)\right\|_{L_{\tau}^{2} L_{\xi}^{2}}
$$

where $S(t):=e^{-\mathrm{i} t \phi(\nabla / \mathrm{i})}$ and $\langle\xi\rangle=\left(1+|\xi|^{2}\right)^{\frac{1}{2}}$.

According to the above definition, it is natural to consider the Fourier restriction space $X_{\tau=P(\xi)}^{s, b}$ for the electric field $\mathcal{E}$ :

$$
\|u\|_{X_{\tau=P(\xi)}^{s, b}}=\left\|e^{\mathrm{itP}(\nabla / \mathrm{i})} u\right\|_{H_{t}^{b} H_{x}^{s}}
$$

with $P(\xi)$ the symmetric $3 \times 3$ matrix given in Section 2.1. However, the above norm is not so convenient in the succeeding linear or nonlinear estimates. Recall that the eigenvalues of $P(\xi)$ are $\lambda_{1}(\xi)=|\xi|^{2}$ and $\lambda_{2}(\xi)=\lambda_{3}(\xi)=\alpha|\xi|^{2}$, and as shown in [12], the norm (2.6) is equivalent to the norm

$$
\|u\|_{X_{S}^{s, b}}=\left\|\langle\xi\rangle^{s}\left\langle\tau+|\xi|^{2}\right\rangle^{b} \tilde{u}(\tau, \xi)\right\|_{L_{\tau}^{2} L_{\xi}^{2}}+\left\|\langle\xi\rangle^{s}\left\langle\tau+\alpha|\xi|^{2}\right\rangle^{b} \tilde{u}(\tau, \xi)\right\|_{L_{\tau}^{2} L_{\xi}^{2}}
$$

Moreover, we will see that the linear or nonlinear estimates for the second part of the norm in (2.7) (i.e., the norm $\left\|\langle\xi\rangle^{s}\left\langle\tau+\alpha|\xi|^{2}\right\rangle^{b} \tilde{u}(\tau, \xi)\right\|_{L_{\tau}^{2} L_{\xi}^{2}}$ ) is essentially the same as the first part of the norm, so without loss of generality, we only need to consider the following Fourier restriction space for the electric field $\mathcal{E}$ :

$$
\|u\|_{X_{S}^{s, b}}=\left\|\langle\xi\rangle^{s}\left\langle\tau+|\xi|^{2}\right\rangle^{b} \tilde{u}(\tau, \xi)\right\|_{L_{\tau}^{2} L_{\xi}^{2}}
$$

That is why we can reduce the argument for the general case $\alpha>1$ to the case $\alpha=1$. 
Note that $\langle\tau+\langle\xi\rangle\rangle \sim\langle\tau+|\xi|\rangle$, so by (2.5) we may then define the resolution space $X_{W}^{s, b}$ for $N$ equipped with the norm

$$
\|u\|_{X_{W}^{s, b}}=\left\|\langle\xi\rangle^{s}\langle\tau+|\xi|\rangle^{b} \tilde{u}(\tau, \xi)\right\|_{L_{\tau}^{2} L_{\xi}^{2}}
$$

For the dissipative equation

$$
u_{t}+(-\Delta)^{\frac{3}{2}} u=f(u), u(0)=u_{0},
$$

where $(-\Delta)^{\frac{3}{2}}$ is defined in $(2.1)$, we define the space $X_{H}^{s, b}$ by analogy with the definition of Bourgain space,

$$
\|u\|_{X_{H}^{s, b}}=\left\|\langle\xi\rangle^{s}\left\langle\mathrm{i} \tau+\xi^{3}\right\rangle^{b} \tilde{u}(\tau, \xi)\right\|_{L_{\tau}^{2} L_{\xi}^{2}} .
$$

$X_{H}^{s, b}$ is the resolution space for the third equation on $\mathcal{B}$ in (2.2). Note that the weight $\left\langle\mathrm{i} \tau+\xi^{3}\right\rangle$ is equivalent to $\left\langle|\tau|+|\xi|^{3}\right\rangle$.

Since in the following we will apply the linear estimates (3.6) and (3.12) (see the next subsection), we shall use the auxiliary spaces $A_{S}^{s}, A_{W}^{s}$, and $A_{H}^{s}$ equipped with the norms

$$
\begin{gathered}
\|u\|_{A_{S}^{s}}=\left\|\langle\xi\rangle^{s}\left\langle\tau+|\xi|^{2}\right\rangle^{-1} \tilde{u}(\tau, \xi)\right\|_{L_{\xi}^{2} L_{\tau}^{1}}, \\
\|u\|_{A_{W}^{s}}=\left\|\langle\xi\rangle^{s}\langle\tau+|\xi|\rangle^{-1} \tilde{u}(\tau, \xi)\right\|_{L_{\xi}^{2} L_{\tau}^{1}} \\
\|u\|_{A_{H}^{s}}=\left\|\langle\xi\rangle^{s}\left\langle|\tau|+|\xi|^{3}\right\rangle^{-1} \tilde{u}(\tau, \xi)\right\|_{L_{\xi}^{2} L_{\tau}^{1}}
\end{gathered}
$$

respectively.

For $T>0$, we define the space $X_{S}^{s, b}(T)$ to be the restriction of distributions in $X_{S}^{s, b}$ to the time interval $[0, T]$ with the induced norm

$$
\|u\|_{X_{S}^{s, b}(T)}=\inf \left\{\|v\|_{X^{s, b}} ; v \in X_{S}^{s, b},\left.v\right|_{[0, T]}=u\right\} .
$$

The spaces $X_{W}^{s, b}(T), X_{H}^{s, b}(T), A_{S}^{s}(T), A_{W}^{s}(T)$, and $A_{H}^{s}(T)$ can be defined in a similar way.

\section{Linear estimates}

To solve (2.2) locally, we introduce a smooth function $\psi(t) \in C_{0}^{\infty}(\mathbb{R})$ satisfying $0 \leq \psi \leq 1, \psi(t)=\psi(-t), \psi(t) \equiv 1$ if $|t| \leq 1$, and $\psi(t) \equiv 0$ if $|t| \geq 2$, and we define $\psi_{T}(t)=$ $\psi(t / T)$. Now we write (2.2)-(2.3) into the following integral form:

$$
\begin{aligned}
& \mathcal{E}=\psi e^{\mathrm{i} t \Delta} \mathcal{E}_{0}+\frac{1}{2} \psi_{T} S_{\mathcal{E}}\left(\left(\psi_{2 T}(N+\bar{N})\right)\left(\psi_{2 T} \mathcal{E}\right)\right)-\mathrm{i} \psi_{T} S_{\mathcal{E}}\left(\left(\psi_{2 T} \mathcal{E}\right) \times\left(\psi_{2 T} \mathcal{B}\right)\right), \\
& N=\psi e^{-\mathrm{i} t\langle\nabla\rangle_{0}} N_{0}-\psi_{T} S_{N}\left(\langle\nabla\rangle^{-1} \Delta\left|\psi_{2 T} \mathcal{E}\right|^{2}\right)-\frac{1}{2} \psi_{T} S_{N}\left(\langle\nabla\rangle^{-1}(N+\bar{N})\right), \\
& \mathcal{B}=\psi e^{-(-\Delta)^{3 / 2}|t|} \mathcal{B}_{0}-\mathrm{i} \psi_{T} \chi_{\mathbb{R}^{+}} S_{\mathcal{B}}\left((-\Delta)^{\frac{1}{2}} \nabla \times\left(\nabla \times\left(\psi_{2 T} \mathcal{E} \times \psi_{2 T} \overline{\mathcal{E}}\right)\right)\right),
\end{aligned}
$$

where the integral operators $S_{\mathcal{E}} f, S_{N} f, S_{\mathcal{B}} f$ are defined as follows:

$$
\begin{aligned}
S_{\mathcal{E}} f & :=\frac{1}{\mathrm{i}} \int_{0}^{t} e^{\mathrm{i}\left(t-t^{\prime}\right) \Delta} f\left(t^{\prime}\right) d t^{\prime}, \\
S_{N} f & :=\frac{1}{\mathrm{i}} \int_{0}^{t} e^{-\mathrm{i}\left(t-t^{\prime}\right)\langle\nabla\rangle} f\left(t^{\prime}\right) d t^{\prime}, \\
S_{\mathcal{B}} f & :=\int_{0}^{t} e^{-\left(t-t^{\prime}\right)(-\Delta)^{3 / 2}} f\left(t^{\prime}\right) d t^{\prime} .
\end{aligned}
$$


We remark that, as in [22], we have extended the linear operator $e^{-(-\Delta)^{3 / 2} t}$ defined on $t \geq 0$ to $e^{-(-\Delta)^{3 / 2}|t|}$ on $t \in \mathbb{R}$ in the integral equation (3.3). We also remark that the purpose of the appearance of $\psi_{2 T}$ in the nonlinear terms is to produce a contraction factor $T^{\epsilon}$ for some $\epsilon>0$, since in our case we will choose the index $b=\frac{1}{2}$, which implies that there will not be any contraction factor in the linear estimate; see Section 4.

In the following, we will show some linear estimates for the linear semigroups and integral operators in (3.1)-(3.3).

For the dispersive equation (2.4), with the definition of $X_{\tau=\phi(\xi)}^{s, b}$, it is easy to see

$$
\left\|\psi(t) S(t) u_{0}\right\|_{X_{\tau=\phi(\xi)}^{s, b}}=\|\psi\|_{H_{t}^{b}}\left\|u_{0}\right\|_{H_{x}^{s}} \lesssim\left\|u_{0}\right\|_{H^{s}} .
$$

And for the integral operators, we have the following basic results (see also [14]).

Proposition 3.1.

(1) Let $s \in \mathbb{R}$. Then there exists $C>0$ such that

$$
\left\|\psi_{T} \int_{0}^{t} S\left(t-t^{\prime}\right) f\left(t^{\prime}\right) d t^{\prime}\right\|_{X_{\tau=\phi(\xi)}^{s, \frac{1}{2}}} \leq C\left(\|f\|_{X_{\tau=\phi(\xi)}^{s,-\frac{1}{2}}}+\|f\|_{\left.A_{\tau=\phi(\xi)}^{s}\right)}\right) .
$$

$$
\begin{gathered}
\text { If } f \in A_{\tau=\phi(\xi)}^{s} \text {, then } \int_{0}^{t} S\left(t-t^{\prime}\right) f\left(t^{\prime}\right) d t^{\prime} \in C\left([-T, T] ; H^{s}\right) \text { for all } T>0 \text {, and } \\
\left\|\int_{0}^{t} S\left(t-t^{\prime}\right) f\left(t^{\prime}\right) d t^{\prime}\right\|_{C\left([-T, T] ; H^{s}\right)} \leq C\|f\|_{A_{\tau=\phi(\xi)}^{s},}
\end{gathered}
$$

where $C$ depends on $T$.

(3) For any $b>0, q \geq 2$ with $b q>1$, there exists $C>0$ depending on $b$ and $q$ such that

$$
\left\|\psi_{T} u\right\|_{X_{\tau=\phi(\xi)}^{s, b}} \leq C T^{-b+\frac{1}{q}}\|u\|_{X_{\tau=\phi(\xi)}^{s, b}}
$$

REMARK 3.1. In the sequel, we take $b=\frac{1}{2}$. Hence if we choose $q>2$ close enough to 2, then the estimate (3.8) implies that the $X^{s, b}$ norm of $\psi_{T} u$ produces a factor $T^{-\epsilon}$ with $\epsilon$ sufficiently small. On the other hand, the positive exponent of $T$ coming from the nonlinear estimate (see Proposition 4.1 and 4.2) can cancel the negative one, hence we can solve (2.2) by contraction methods.

Now we are going to give some linear estimates for the dissipative equation (2.10) in Propositions 3.4-3.6 below. To this purpose, first we give two general lemmas, following which we can easily prove Propositions 3.4 and 3.5. Although we are inspired by L. Molinet and F. Ribaud [22], and most of the ideas is essentially contained in [22], for the reader's convenience we give a direct proof in Appendix A.

Lemma 3.2. If $\varphi(\xi) \in C\left(\mathbb{R}^{d}\right), \varphi(\xi) \geq 0$, and $\varphi(\xi)>0$ when $\xi \neq 0$, then

$$
\left\|\langle|\tau|+\varphi(\xi)\rangle^{1 / 2} \mathscr{F}_{t}\left(\psi(t) e^{-|t| \varphi(\xi)}\right)\right\|_{L_{\tau}^{2}} \leq C,
$$

where $C$ is independent of $\xi$.

Lemma 3.3. If $\varphi(\xi) \in C\left(\mathbb{R}^{d}\right), \varphi(\xi) \geq 0$, and $\varphi(\xi)>0$ when $\xi \neq 0$, then we have

$$
\left\|\langle\mathrm{i} \tau+\varphi(\xi)\rangle^{1 / 2} \mathscr{F}_{t}(g(\xi, t))\right\|_{L_{\tau}^{2}}^{2} \leq C\left[\left(\int_{\mathbb{R}} \frac{|\hat{f}(\tau, \xi)|}{\langle\mathrm{i} \tau+\varphi(\xi)\rangle} d \tau\right)^{2}+\int_{\mathbb{R}} \frac{|\hat{f}(\tau, \xi)|^{2}}{\langle\mathrm{i} \tau+\varphi(\xi)\rangle} d \tau\right]
$$


where $g(\xi, t)=\psi(t) \int_{\mathbb{R}} \frac{e^{\mathrm{i} t \tau}-e^{-\varphi(\xi)|t|}}{\mathbf{i} \tau+\varphi(\xi)} \hat{f}(\tau, \xi) d \tau$

Now we return to our dissipative equation (2.10). By Lemma 3.2, the free term can be estimated easily.

Proposition 3.4. Let $s \in \mathbb{R}$. There exists $C>0$ such that

$$
\left\|\psi(t) e^{-(-\Delta)^{3 / 2}|t|} u_{0}\right\|_{X_{H}^{s, \frac{1}{2}}} \leq C\left\|u_{0}\right\|_{H^{s}}, \forall u_{0} \in H^{s}\left(\mathbb{R}^{3}\right) .
$$

Proof. From the definition of $X_{H}^{s, \frac{1}{2}}$ and Lemma $3.2\left(\right.$ set $\left.\varphi(\xi)=|\xi|^{3}\right)$, one sees

$$
\begin{aligned}
\left\|\psi(t) e^{-(-\Delta)^{3 / 2}|t|} u_{0}\right\|_{X_{H}^{s, \frac{1}{2}}} & \leq\left\|\langle\xi\rangle^{s} \hat{u}_{0}(\xi)\right\|\left\langle|\tau|+|\xi|^{3}\right\rangle^{\frac{1}{2}} \mathscr{F}_{t}\left(\psi(t) e^{-|t||\xi|^{3}}\right)(\tau)\left\|_{L_{\tau}^{2}}\right\|_{L_{\xi}^{2}\left(\mathbb{R}^{3}\right)} \\
& \leq C\left\|\langle\xi\rangle^{s} \hat{u}_{0}\right\|_{L_{\xi}^{2}\left(\mathbb{R}^{3}\right)}
\end{aligned}
$$

which completes the proof of (3.11) as desired.

Based on Lemma 3.3, the linear estimate for the Duhamel term is given by the following Proposition.

Proposition 3.5. If $s \in \mathbb{R}, t>0$, then there exists $C>0$ such that

$$
\left\|\chi_{\mathbb{R}^{+}}(t) \psi(t) \int_{0}^{t} e^{-(-\Delta)^{3 / 2}\left(t-t^{\prime}\right)} f\left(t^{\prime}\right) d t^{\prime}\right\|_{X_{H}^{s, \frac{1}{2}}} \lesssim\|f\|_{X_{H}^{s,-\frac{1}{2}}}+\|f\|_{A_{H}^{s}}
$$

Proof. Note that

$$
\begin{aligned}
& \mathscr{F}_{x}\left(\chi_{\mathbb{R}^{+}}(t) \psi(t) \int_{0}^{t} e^{-(-\Delta)^{3 / 2}\left(t-t^{\prime}\right)} f\left(t^{\prime}\right) d t^{\prime}\right) \\
= & \chi_{\mathbb{R}^{+}}(t) \psi(t) \int_{0}^{t} e^{-|\xi|^{3}\left(t-t^{\prime}\right)} \hat{f}\left(t^{\prime}, \xi\right) d t^{\prime} \\
= & C \chi_{\mathbb{R}^{+}}(t) \psi(t) e^{-|\xi|^{3} t} \int_{0}^{t} \int_{\mathbb{R}^{3}} e^{\left(|\xi|^{3}+\mathrm{i} \tau^{\prime}\right) t^{\prime}} \tilde{f}\left(\tau^{\prime}, \xi\right) d \tau^{\prime} d t^{\prime} \\
= & C \chi_{\mathbb{R}^{+}}(t) \psi(t) \int_{\mathbb{R}} \frac{e^{i \tau^{\prime} t}-e^{-|\xi|^{3} t}}{\mathrm{i} \tau^{\prime}+|\xi|^{3}} \tilde{f}\left(\tau^{\prime}, \xi\right) d \tau^{\prime},
\end{aligned}
$$

where we have used Fubini's theorem in the last step. Note also that

$$
\left\|\chi_{\mathbb{R}^{+}}(t) f(t)\right\|_{H^{\frac{1}{2}}(\mathbb{R})} \lesssim\|f\|_{H^{\frac{1}{2}(\mathbb{R})}} .
$$

Combining the above two estimates with Lemma 3.3, we can easily obtain (3.12) as desired.

In the paper, we will use the linear estimate (3.11) and (3.12) for the integral equation (3.3). Since the embedding $X_{H}^{s, \frac{1}{2}} \hookrightarrow C_{t}^{0} H_{x}^{s}$ no longer holds, a natural question is whether the solution of $(2.10)$ can be a continuous flow in $H^{s}$ provided that we know $f \in A_{H}^{s}$. The following proposition gives a definite answer to this problem.

Proposition 3.6. If $s \in \mathbb{R}, t>0, f \in A_{H}^{s}$, then for any $T>0$ we have

$$
\int_{0}^{t} e^{-(-\Delta)^{3 / 2}\left(t-t^{\prime}\right)} f\left(t^{\prime}\right) d t^{\prime} \in C\left([0, T] ; H^{s}\right) .
$$


Moreover, there exists $C>0$ depending on $T$ such that

$$
\left\|\int_{0}^{t} e^{-(-\Delta)^{3 / 2}\left(t-t^{\prime}\right)} f\left(t^{\prime}\right) d t^{\prime}\right\|_{C\left([0, T] ; H^{s}\right)} \leq C\|f\|_{A_{H}^{s}}
$$

Proof. Without loss of generality, we may assume $s=0$. First we prove (3.13). By Plancherel's identity, it is sufficient to show

$$
\int_{0}^{t} e^{-|\xi|^{3}\left(t-t^{\prime}\right)} \hat{f}\left(t^{\prime}, \xi\right) d t^{\prime} \in C\left([0, T] ; L_{\xi}^{2}\right) .
$$

Setting $F(t):=\int_{0}^{t} e^{-|\xi|^{3}\left(t-t^{\prime}\right)} \hat{f}\left(t^{\prime}, \xi\right) d t^{\prime}$, we want to prove

$$
\left\|F\left(t_{1}\right)-F\left(t_{2}\right)\right\|_{L_{\xi}^{2}} \rightarrow 0, \quad \text { as }\left|t_{1}-t_{2}\right| \rightarrow 0, t_{1}, t_{2} \in[0, T] .
$$

By applying Lebesgue's dominated convergence theorem, we can then obtain (3.13) with $s=0$ as desired.

Using Fubini's theorem, one has

$$
F(t)=\int_{0}^{t} e^{-|\xi|^{3}\left(t-t^{\prime}\right)} \int_{\mathbb{R}} e^{i t^{\prime} \tau} \tilde{f}(\tau, \xi) d \tau d t^{\prime}=\int_{\mathbb{R}} \tilde{f}(\tau, \xi) \frac{e^{\mathrm{i} t \tau}-e^{-|\xi|^{3} t}}{\mathrm{i} \tau+|\xi|^{3}} d \tau .
$$

Assume $a_{\xi}(t, \tau):=e^{\mathrm{i} t \tau}-e^{-|\xi|^{3} t}$, and let $t_{1}, t_{2} \in[0, T]$ with $\left|t_{1}-t_{2}\right| \ll 1$. Then

$$
\begin{aligned}
& \left\|F\left(t_{1}\right)-F\left(t_{2}\right)\right\|_{L_{\xi}^{2}}^{2} \\
= & \int_{\mathbb{R}^{3}} \int_{\mathbb{R}} \int_{\mathbb{R}} \frac{a_{\xi}\left(t_{1}, \tau\right)-a_{\xi}\left(t_{2}, \tau\right)}{\mathrm{i} \tau+|\xi|^{3}} \cdot \frac{\bar{a}_{\xi}\left(t_{1}, \tau^{\prime}\right)-\bar{a}_{\xi}\left(t_{2}, \tau^{\prime}\right)}{-\mathrm{i} \tau^{\prime}+|\xi|^{3}} \tilde{f}(\tau, \xi) \overline{\tilde{f}}\left(\tau^{\prime}, \xi\right) d \tau d \tau^{\prime} d \xi
\end{aligned}
$$

On one hand, the integrand in the above equality tends to zero as $\left|t_{1}-t_{2}\right| \rightarrow 0$ for fixed $\tau, \tau^{\prime}$, and $\xi$. On the other hand, since $\left.\left.|\mathrm{i} \tau+| \xi\right|^{3}|\sim| \tau|+| \xi\right|^{3}$, we have

$$
\left|\frac{a_{\xi}\left(t_{1}, \tau\right)-a_{\xi}\left(t_{2}, \tau\right)}{\mathrm{i} \tau+|\xi|^{3}}\right| \leq\left\{\begin{array}{cc}
2\left|t_{1}-t_{2}\right|, & \text { if }|\tau|+|\xi|^{3} \leq 1 \\
\frac{4}{|\tau|+|\xi|^{3}}, & \text { if }|\tau|+|\xi|^{3}>1
\end{array} \leq C\left\langle|\tau|+|\xi|^{3}\right\rangle^{-1}\right.
$$

so the integrand in (3.16) can be controlled by

$$
C\left\langle|\tau|+|\xi|^{3}\right\rangle^{-1} \tilde{f}(\tau, \xi)\left\langle\left|\tau^{\prime}\right|+|\xi|^{3}\right\rangle^{-1} \overline{\tilde{f}}\left(\tau^{\prime}, \xi\right),
$$

which belongs to $L_{\xi}^{1} L_{\tau}^{1} L_{\tau^{\prime}}^{1}$ since $f \in A_{H}^{0}$. We therefore obtain (3.15), which in turn gives (3.13) with $s=0$.

To prove (3.14), we take $t_{2}=0$ (note that $F(0)=0$ ). Arguing as above, we can obtain

$$
\|F(t)\|_{L_{\xi}^{2}} \leq C\|f\|_{A_{H}^{0}}
$$

for $0 \leq t \leq \frac{1}{2}$. Then using a step by step argument, one gets (3.14) with $C$ depending on $T$. 


\section{Bilinear estimates}

The main aim of this section is to give bilinear estimates for $\mathcal{E}$ and $\mathcal{B}$. Since the system (2.2) is the classical Zakharov system

$$
\left\{\begin{array}{l}
\mathrm{i} \mathcal{E}_{t}+\Delta \mathcal{E}-n \mathcal{E}=0 \\
n_{t t}-\Delta n=\Delta|\mathcal{E}|^{2}
\end{array}\right.
$$

coupled to a given magnetic field $\mathcal{B}$ by an additional term $\mathrm{i} \mathcal{E} \times \mathcal{B}$ in the first equation of (2.2), the following bilinear estimates for $\mathcal{E}$ and $N$ are essentially proved in [14].

Proposition 4.1. Suppose $k$ and $l$ satisfy

$$
l \leq k \leq l+1, \frac{1}{2}(l+1) \leq k, l \geq 0 .
$$

Moreover, assume $\mathcal{E}$ and $N$ have compact support in time $|t| \leq C T$ with $T \leq 1$. Then there exists $\theta_{1}>0$ such that

$$
\begin{aligned}
\|N E\|_{X_{S}^{k,-\frac{1}{2}}(T)}+\|\bar{N} E\|_{X_{S}^{k,-\frac{1}{2}}(T)} & \lesssim T^{\theta_{1}}\|N\|_{X_{W}^{l, \frac{1}{2}}(T)}\|E\|_{X_{S}^{k, \frac{1}{2}}(T)}, \\
\|E \cdot \bar{E}\|_{X_{W}^{l+1,-\frac{1}{2}}(T)} & \lesssim T^{\theta_{1}}\|E\|_{X_{S}^{k, \frac{1}{2}}(T)}^{2}, \\
\|N E\|_{A_{S}^{k}(T)}+\|\bar{N} E\|_{A_{S}^{k}(T)} & \lesssim T^{\theta_{1}}\|N\|_{X_{W}^{l, \frac{1}{2}}(T)}\|E\|_{X_{S}^{k, \frac{1}{2}}(T)}, \\
\|E \cdot \bar{E}\|_{A_{W}^{l+1}(T)} & \lesssim T^{\theta_{1}}\|E\|_{X_{S}^{k, \frac{1}{2}}(T)}^{2},
\end{aligned}
$$

where $E$ denotes any component of $\mathcal{E}=\left(\mathcal{E}_{1}, \mathcal{E}_{2}, \mathcal{E}_{3}\right)$.

So we mainly deal with the bilinear estimates for $\mathcal{E}$ and $\mathcal{B}$ in this section. The main results are stated as follows.

Proposition 4.2. Assume $k>\frac{1}{2}$, and $\mathcal{E}$ and $\mathcal{B}$ have compact support in time $0 \leq t \leq$ $C T$ with $T \leq 1$. Then there exists $\theta_{2}>0$ such that

$$
\begin{aligned}
\|B E\|_{X_{S}^{k,-\frac{1}{2}}(T)} & \lesssim T^{\theta_{2}}\|B\|_{X_{H}^{k-\frac{3}{2}, \frac{1}{2}}(T)}\|E\|_{X_{S}^{k, \frac{1}{2}}(T)}, \\
\|E \bar{F}\|_{X_{H}^{k+\frac{3}{2},-\frac{1}{2}}(T)} & \lesssim T^{\theta_{2}}\|E\|_{X_{S}^{k, \frac{1}{2}}(T)}\|F\|_{X_{S}^{k, \frac{1}{2}}(T)}, \\
\|B E\|_{A_{S}^{k}(T)} & \lesssim T^{\theta_{2}}\|B\|_{X_{H}^{k-\frac{3}{2}, \frac{1}{2}}(T)}\|E\|_{X_{S}^{k, \frac{1}{2}}(T)}, \\
\|E \bar{F}\|_{A_{H}^{k+\frac{3}{2}}(T)} & \lesssim T^{\theta_{2}}\|E\|_{X_{S}^{k, \frac{1}{2}}(T)}\|F\|_{X_{S}^{k, \frac{1}{2}}(T)},
\end{aligned}
$$

where $E$ or $F$ denotes any component of $\mathcal{E}=\left(\mathcal{E}_{1}, \mathcal{E}_{2}, \mathcal{E}_{3}\right)$, and $B$ denotes any component of $\mathcal{B}=\left(\mathcal{B}_{1}, \mathcal{B}_{2}, \mathcal{B}_{3}\right)$.

Inspired by the proof of Proposition 4.1 in [14], in order to prove Proposition 4.2, we first give some lemmas. In Lemmas $4.3-4.6$ below, we set $\delta(r):=3\left(\frac{1}{2}-\frac{1}{r}\right)$ with $2 \leq r \leq 6$.

Lemma 4.3. Let $b_{0}>\frac{1}{2}, 0 \leq b \leq b_{0}$. Then we have

$$
\left\|\mathscr{F}^{-1}\left(\left\langle\tau+|\xi|^{2}\right\rangle^{-b} \tilde{u}(\tau, \xi)\right)\right\|_{L_{t}^{q} L_{x}^{r}} \lesssim\|u\|_{L_{t, x}^{2}}
$$

with

$$
\frac{2}{q}=1-\eta \frac{b}{b_{0}}, \delta(r)=(1-\eta) \frac{b}{b_{0}},
$$


where $0 \leq \eta \leq 1$.

Lemma 4.3 follows by interpolation between the trivial case

$$
\|f\|_{L_{t}^{2} L_{x}^{2}}=\|f\|_{X_{S}^{0,0}}
$$

and the Strichartz inequality of the Schrödinger equation:

$$
\|f\|_{L_{t}^{\frac{2}{1-\eta}} L_{x}^{r_{0}}} \lesssim\|f\|_{X_{S}^{0, b_{0}}}, \delta\left(r_{0}\right)=1-\eta .
$$

In the estimate (4.7), there is no factor $T^{\theta}$ produced, which is necessary for our contraction argument, so we will prove a stronger version of (4.7).

Lemma 4.4. Let $b_{0}>\frac{1}{2}, 0 \leq a, \kappa \leq 1$, and $(1-\kappa) a \leq b_{0}$. Moreover, assume that $u$ has compact support in time $0 \leq t \leq C T$ with $T \leq 1$. Then there holds

$$
\left\|\mathscr{F}^{-1}\left(\left\langle\tau+|\xi|^{2}\right\rangle^{-a} \tilde{u}(\tau, \xi)\right)\right\|_{L_{t}^{q} L_{x}^{r}} \lesssim T^{\frac{\kappa a}{2}}\|u\|_{L_{t . x}^{2}},
$$

with

$$
\frac{2}{q}=1-\eta \frac{(1-\kappa) a}{b_{0}}, \delta(r)=(1-\eta) \frac{(1-\kappa) a}{b_{0}}
$$

where $0 \leq \eta \leq 1$.

Proof. Using the estimate (4.7) with $b=(1-\kappa) a$, by Hölder's inequality we have

$$
\begin{aligned}
\left\|\mathscr{F}^{-1}\left(\left\langle\tau+|\xi|^{2}\right\rangle^{-a} \tilde{u}(\tau, \xi)\right)\right\|_{L_{t}^{q} L_{x}^{2}} & \lesssim\left\|\left\langle\tau+|\xi|^{2}\right\rangle^{-\kappa a} \tilde{u}(\tau, \xi)\right\|_{L_{\tau}^{2} L_{\xi}^{2}} \\
& \lesssim\|u\|_{L_{t, x}^{2}}^{1-\kappa}\left\|\left\langle\tau+|\xi|^{2}\right\rangle^{-a} \tilde{u}(\tau, \xi)\right\|_{L_{\tau, \xi}^{2}}^{\kappa}
\end{aligned}
$$

Because $u$ has compact support in time $0 \leq t \leq C T$, one can see that

$$
\left\|\left\langle\tau+|\xi|^{2}\right\rangle^{-a} \tilde{u}(\tau, \xi)\right\|_{L_{\tau, \xi}^{2}}=\left\|\left\langle\partial_{t}\right\rangle^{-a} e^{-i t \Delta} u\right\|_{L_{t, x}^{2}} \lesssim T^{\frac{1}{2}-\frac{1}{p}}\left\|\left\langle\partial_{t}\right\rangle^{-a} u\right\|_{L_{x}^{2} L_{t}^{p}} .
$$

Note that $H^{a}(\mathbb{R}) \hookrightarrow L^{p}(\mathbb{R})$ with $a=\frac{1}{2}-\frac{1}{p}$ if $0 \leq a<\frac{1}{2}, H^{a}(\mathbb{R}) \hookrightarrow L^{p}(\mathbb{R})$ with $2 \leq p<\infty$ if $a=\frac{1}{2}$ (in particular, we choose $p=4$ ), and $H^{a}(\mathbb{R}) \hookrightarrow L^{\infty}(\mathbb{R})$ if $a>\frac{1}{2}$, so we deduce from the above inequality together with the assumptions $T \leq 1$ and $0 \leq a \leq 1$ that

$$
\left\|\left\langle\tau+|\xi|^{2}\right\rangle^{-a} \tilde{u}(\tau, \xi)\right\|_{L_{\tau, \xi}^{2}} \lesssim T^{\frac{1}{2} a}\|u\|_{L_{t, x}^{2}} .
$$

Inserting this estimate into (4.10), we thus obtain (4.9).

Note that when $\kappa=0$, the estimate (4.9) reduces to (4.7). Indeed, in the sequel, we will choose $\kappa>0$ sufficiently small, and we will see the factor $T^{\theta_{2}}$ in the estimates (4.3)-(4.6) comes from $E$, as in the estimate (4.9).

Lemmas 4.3 and 4.4 deal with the Schrödinger operator i $\partial_{t}+\Delta$, and one can also obtain similar results for the dissipative operator $\partial_{t}+(-\Delta)^{\frac{3}{2}}$. However, the following Lemma 4.5 is enough for our further arguments, since all the non-linear terms in (4.3)-(4.6) contain $E$, from which we can get the factor $T^{\theta_{2}}$.

Lemma 4.5. Let $b_{0}>\frac{1}{2}, 0 \leq b \leq b_{0}$, and $\frac{2}{q}=1-\frac{b}{b_{0}}$. Then

$$
\left\|\mathscr{F}^{-1}\left(\left\langle|\tau|+|\xi|^{3}\right\rangle^{-b} \tilde{u}(\tau, \xi)\right)\right\|_{L_{t}^{q} L_{x}^{2}} \lesssim\|u\|_{L_{t, x}^{2}} .
$$


Interpolation between (4.8) and the estimate $\|f\|_{L^{\infty} L^{2}} \lesssim\|f\|_{X_{H}^{0, b_{0}}}$ immediately gives (4.11).

LEMma 4.6. Let $b_{0}>\frac{1}{2}, 0 \leq \kappa, a_{1}, a_{2}, a_{3} \leq 1$, and

$$
\max \left\{a_{1}, a_{2}, a_{3}\right\} \leq b_{0}<(1-\kappa)\left(a_{1}+a_{2}\right)+a_{3} .
$$

Suppose $s_{1}$ and $s_{2}$ satisfy

$$
s_{1} \geq \frac{5}{2}-\frac{(1-\kappa)\left(a_{1}+a_{2}\right)+a_{3}}{b_{0}} \geq 0, s_{2}=0,
$$

or

$$
s_{2} \geq \frac{5}{2}-\frac{(1-\kappa)\left(a_{1}+a_{2}\right)+a_{3}}{b_{0}} \geq 0, s_{1}=0 .
$$

Let $u_{1}, u_{2}, u_{3} \in L^{2}$ be such that they all have compact support in time $[0, C T]$ with $T \leq 1$. Then

$$
\int \frac{\left|\tilde{u}_{1}\left(\tau_{1}, \xi_{1}\right) \tilde{u}_{2}\left(\tau_{2}, \xi_{2}\right) \tilde{u}_{3}\left(\tau_{3}, \xi_{3}\right)\right|}{\left\langle\sigma_{1}\right\rangle^{a_{1}}\left\langle\sigma_{2}\right\rangle^{a_{2}}\left\langle\sigma_{3}\right\rangle^{a_{3}}\left\langle\xi_{2}\right\rangle^{s_{1}}\left\langle\xi_{3}\right\rangle^{s_{2}}} \lesssim T^{\theta}\left\|u_{1}\right\|_{L^{2}}\left\|u_{2}\right\|_{L^{2}}\left\|u_{3}\right\|_{L^{2}}
$$

where $\sigma_{i}=\tau_{i}+\left|\xi_{i}\right|^{2}, i=1,2, \sigma_{3}=\left|\tau_{3}\right|+\left|\xi_{3}\right|^{3},\left(\tau_{1}, \xi_{1}\right)=\left(\tau_{2}, \xi_{2}\right)+\left(\tau_{3}, \xi_{3}\right)$, and $\theta=\frac{\kappa}{2}\left(a_{1}+\right.$ $\left.a_{2}\right)$. that

Proof. First, we consider the case $s_{2}=0$. By Hölder's inequality, one can see

$$
\begin{gathered}
\int \frac{\left|\tilde{u}_{1} \tilde{u}_{2} \tilde{u}_{3}\right|}{\left\langle\sigma_{1}\right\rangle^{a_{1}}\left\langle\sigma_{2}\right\rangle^{a_{2}}\left\langle\sigma_{3}\right\rangle^{a_{3}}\left\langle\xi_{2}\right\rangle^{s_{1}}} \lesssim\left\|\mathscr{F}^{-1}\left(\left\langle\sigma_{1}\right\rangle^{-a_{1}}\left|\tilde{u}_{1}\right|\right)\right\|_{L^{q_{1}} L^{r_{1}}}\left\|\mathscr{F}^{-1}\left(\left\langle\sigma_{2}\right\rangle^{-a_{2}}\left\langle\xi_{2}\right\rangle^{-s_{1}}\left|\tilde{u}_{2}\right|\right)\right\|_{L^{q_{2}} L^{r_{2}}} \\
\cdot\left\|\mathscr{F}^{-1}\left(\left\langle\sigma_{3}\right\rangle^{-a_{3}}\left|\tilde{u}_{3}\right|\right)\right\|_{L^{q_{3}} L^{2}},
\end{gathered}
$$

where

$$
\begin{aligned}
& \frac{1}{q_{1}}+\frac{1}{q_{2}}+\frac{1}{q_{3}}=1, \\
& \frac{1}{r_{1}}+\frac{1}{r_{2}}+\frac{1}{2}=1 .
\end{aligned}
$$

From Lemmas 4.4-4.5, we have

$$
\begin{aligned}
\left\|\mathscr{F}^{-1}\left(\left\langle\sigma_{1}\right\rangle^{-a_{1}}\left|\tilde{u}_{1}\right|\right)\right\|_{L^{q_{1}} L^{r_{1}}} & \lesssim T^{\frac{1}{2} \kappa a_{1}}\left\|u_{1}\right\|_{L^{2}}, \\
\left\|\mathscr{F}^{-1}\left(\left\langle\sigma_{3}\right\rangle^{-a_{3}}\left|\tilde{u}_{3}\right|\right)\right\|_{L^{q_{3}} L^{2}} & \lesssim\left\|u_{3}\right\|_{L^{2}},
\end{aligned}
$$

with

$$
\frac{2}{q_{1}}=1-\eta \frac{(1-\kappa) a_{1}}{b_{0}}, \delta\left(r_{1}\right)=(1-\eta) \frac{(1-\kappa) a_{1}}{b_{0}}
$$

and

$$
\frac{2}{q_{3}}=1-\frac{a_{3}}{b_{0}}
$$

Note that

$$
\left\|\mathscr{F}^{-1}\left(\left\langle\xi_{2}\right\rangle^{-s_{1}}\left\langle\sigma_{2}\right\rangle^{-a_{2}}\left|\tilde{u}_{2}\right|\right)\right\|_{L^{q_{2} L^{r_{2}}}} \lesssim\left\|\mathscr{F}^{-1}\left(\left\langle\sigma_{2}\right\rangle^{-a_{2}}\left|\tilde{u}_{2}\right|\right)\right\|_{L^{q_{2} L^{r}}} \lesssim T^{\frac{\kappa a_{2}}{2}}\left\|u_{2}\right\|_{L^{2}}
$$


where we have used the embedding $H^{s_{1}, r}\left(\mathbb{R}^{3}\right) \hookrightarrow L^{r_{2}}\left(\mathbb{R}^{3}\right)$ and the estimate (4.9) in the first and second step respectively, and the parameters should satisfy

$$
\begin{aligned}
& \frac{2}{q_{2}}=1-\eta \frac{(1-\kappa) a_{2}}{b_{0}}, \delta(r)=(1-\eta) \frac{(1-\kappa) a_{2}}{b_{0}}, \\
& s_{1} \geq 3\left(\frac{1}{r}-\frac{1}{r_{2}}\right) \geq 0, r_{2}<\infty .
\end{aligned}
$$

Now (4.16), (4.20), (4.21), and (4.23) give $b_{0}=\eta(1-\kappa)\left(a_{1}+a_{2}\right)+a_{3}$, and (4.17), (4.20), (4.23), and (4.24) give $s_{1} \geq \frac{5}{2}-\frac{(1-\kappa)\left(a_{1}+a_{2}\right)+a_{3}}{b_{0}} \geq 0$. It is then easy to see that these restrictions hold under the conditions (4.12) and (4.13). Hence, the estimate (4.14) follows from (4.15), (4.18), (4.19), and (4.22).

The case $s_{1}=0$ can be treated in a similar way, hence we omit further details. This ends the proof of Lemma 4.6.

Proof of Proposition 4.2. In order to unify the variables, let $\tilde{B}=\tilde{B}\left(\tau_{3}, \xi_{3}\right)$, let the variables for $\tilde{\bar{F}}$ be $-\tau_{2}$ and $-\xi_{2}$, and set $\widetilde{B E}=(\tilde{B} * \tilde{E})\left(\tau_{1}, \xi_{1}\right), \widetilde{E \bar{F}}=(\tilde{E} * \tilde{\bar{F}})\left(\tau_{3}, \xi_{3}\right)$. With such notations, in order to prove (4.3)-(4.6), by a duality argument and some change of variables, it suffices to show

$$
\begin{aligned}
I & :=\int_{*} \frac{\left|\tilde{u}_{1}\left(\tau_{1}, \xi_{1}\right) \tilde{u}_{2}\left(\tau_{2}, \xi_{2}\right) \tilde{u}_{3}\left(\tau_{3}, \xi_{3}\right)\right|\left\langle\xi_{1}\right\rangle^{k}}{\left\langle\sigma_{1}\right\rangle^{1 / 2}\left\langle\sigma_{2}\right\rangle^{1 / 2}\left\langle\sigma_{3}\right\rangle^{1 / 2}\left\langle\xi_{2}\right\rangle^{k}\left\langle\xi_{3}\right\rangle^{k-3 / 2}} \lesssim T^{\mu}\left\|u_{1}\right\|_{L^{2}}\left\|u_{2}\right\|_{L^{2}}\left\|u_{3}\right\|_{L^{2}}, \\
J & :=\int_{*} \frac{\left|\tilde{u}_{1}\left(\tau_{1}, \xi_{1}\right) \tilde{u}_{2}\left(\tau_{2}, \xi_{2}\right) \tilde{u}_{3}\left(\tau_{3}, \xi_{3}\right)\right|\left\langle\xi_{3}\right\rangle^{k+3 / 2}}{\left\langle\sigma_{1}\right\rangle^{1 / 2}\left\langle\sigma_{2}\right\rangle^{1 / 2}\left\langle\sigma_{3}\right\rangle^{1 / 2}\left\langle\xi_{1}\right\rangle^{k}\left\langle\xi_{2}\right\rangle^{k}} \lesssim T^{\mu}\left\|u_{1}\right\|_{L^{2}}\left\|u_{2}\right\|_{L^{2}}\left\|u_{3}\right\|_{L^{2}}, \\
K & :=\int_{*} \frac{\left|\hat{w}_{1}\left(\xi_{1}\right) \tilde{u}_{2}\left(\tau_{2}, \xi_{2}\right) \tilde{u}_{3}\left(\tau_{3}, \xi_{3}\right)\right|\left\langle\xi_{1}\right\rangle^{k}}{\left\langle\sigma_{1}\right\rangle\left\langle\sigma_{2}\right\rangle^{1 / 2}\left\langle\sigma_{3}\right\rangle^{1 / 2}\left\langle\xi_{2}\right\rangle^{k}\left\langle\xi_{3}\right\rangle^{k-3 / 2}} \lesssim T^{\mu}\left\|w_{1}\right\|_{L^{2}}\left\|u_{2}\right\|_{L^{2}}\left\|u_{3}\right\|_{L^{2}}, \\
L & :=\int_{*} \frac{\left|\tilde{u}_{1}\left(\tau_{1}, \xi_{1}\right) \tilde{u}_{2}\left(\tau_{2}, \xi_{2}\right) \hat{w}_{3}\left(\xi_{3}\right)\right|\left\langle\xi_{3}\right\rangle^{k+3 / 2}}{\left\langle\sigma_{1}\right\rangle^{1 / 2}\left\langle\sigma_{2}\right\rangle^{1 / 2}\left\langle\sigma_{3}\right\rangle\left\langle\xi_{1}\right\rangle^{k}\left\langle\xi_{2}\right\rangle^{k}} \lesssim T^{\mu}\left\|u_{1}\right\|_{L^{2}}\left\|u_{2}\right\|_{L^{2}}\left\|w_{3}\right\|_{L^{2}},
\end{aligned}
$$

for all $u_{1}, u_{2}, u_{3} \in L_{t, x}^{2}$ and $w_{1}, w_{3} \in L_{x}^{2}$, where $*$ denotes the restriction

$$
\begin{aligned}
& \left(\tau_{1}, \xi_{1}\right)=\left(\tau_{2}, \xi_{2}\right)+\left(\tau_{3}, \xi_{3}\right), \\
& \sigma_{i}=\tau_{i}+\left|\xi_{i}\right|^{2}, i=1,2, \sigma_{3}=\left|\tau_{3}\right|+\left|\xi_{3}\right|^{3} .
\end{aligned}
$$

In the following, we will prove the estimates (4.25)-(4.28) respectively.

Proof of (4.25). We consider the following two cases $I_{1}$ and $I_{2}$ :

Case 1: $\left|\xi_{1}\right| \lesssim\left|\xi_{2}\right|$. Note that $\sigma_{3}=\left|\tau_{3}\right|+\left|\xi_{3}\right|^{3}$, so $\left\langle\xi_{3}\right\rangle \leq\left\langle\sigma_{3}\right\rangle^{\frac{1}{3}}$. Then the contribution of this case to $I$ is estimated by

$$
I_{1} \lesssim \int \frac{\left|\tilde{u}_{1} \tilde{u}_{2} \tilde{u}_{3}\right|}{\left\langle\sigma_{1}\right\rangle^{1 / 2}\left\langle\sigma_{2}\right\rangle^{1 / 2}\left\langle\sigma_{3}\right\rangle^{1 / 2}\left\langle\xi_{3}\right\rangle^{k-3 / 2}} \lesssim \int \frac{\left|\tilde{u}_{1} \tilde{u}_{2} \tilde{u}_{3}\right|}{\left\langle\sigma_{1}\right\rangle^{1 / 2}\left\langle\sigma_{2}\right\rangle^{1 / 2}\left\langle\xi_{3}\right\rangle^{k}} .
$$

Case 2: $\left|\xi_{1}\right| \gg\left|\xi_{2}\right|$. This case implies $\left|\xi_{1}\right| \sim\left|\xi_{3}\right|$, so the contribution of this case to $I$ is estimated by

$$
I_{2} \lesssim \int \frac{\left|\tilde{u}_{1} \tilde{u}_{2} \tilde{u}_{3}\right|\left\langle\xi_{3}\right\rangle^{3 / 2}}{\left\langle\sigma_{1}\right\rangle^{1 / 2}\left\langle\sigma_{2}\right\rangle^{1 / 2}\left\langle\sigma_{3}\right\rangle^{1 / 2}\left\langle\xi_{2}\right\rangle^{k}} \lesssim \int \frac{\left|\tilde{u}_{1} \tilde{u}_{2} \tilde{u}_{3}\right|}{\left\langle\sigma_{1}\right\rangle^{1 / 2}\left\langle\sigma_{2}\right\rangle^{1 / 2}\left\langle\xi_{2}\right\rangle^{k}}
$$

Letting $b_{0}=1 / 2+\epsilon$, we apply Lemma 4.6 with $a_{1}=a_{2}=\frac{1}{2}, a_{3}=0$, and $s_{1}=k, s_{2}=$ 0 or $s_{1}=0, s_{2}=k$, and we see that

$$
I_{1}+I_{2} \lesssim T^{\frac{1}{2} \kappa}\left\|u_{1}\right\|_{L^{2}}\left\|u_{2}\right\|_{L^{2}}\left\|u_{3}\right\|_{L^{2}}
$$


provided that $k, \kappa$, and $\epsilon$ satisfy

$$
\begin{aligned}
& \frac{1}{2}+\epsilon<1-\kappa, \\
& k \geq \frac{5}{2}-\frac{1-\kappa}{1 / 2+\epsilon} \geq 0 .
\end{aligned}
$$

If $k>\frac{1}{2}$, then we can always choose $\epsilon$ and $\kappa$ sufficiently small such that the conditions (4.30)-(4.31) hold. Hence, we get the estimate (4.25), which in turn gives (4.3).

Proof of (4.26). Similar to the proof of (4.25), we set $b_{0}=\frac{1}{2}+\epsilon$ again, where $\epsilon>0$ is small and will be chosen later. We split the integral into three cases.

Case 1: $\left|\xi_{1}\right| \gg\left|\xi_{2}\right|$. The contribution of this case to $J$ is estimated by

$$
J_{1} \lesssim \int \frac{\left|\tilde{u}_{1} \tilde{u}_{2} \tilde{u}_{3}\right|\left\langle\xi_{3}\right\rangle^{3 / 2}}{\left\langle\sigma_{1}\right\rangle^{1 / 2}\left\langle\sigma_{2}\right\rangle^{1 / 2}\left\langle\sigma_{3}\right\rangle^{1 / 2}\left\langle\xi_{2}\right\rangle^{k}} \lesssim \int \frac{\left|\tilde{u}_{1} \tilde{u}_{2} \tilde{u}_{3}\right|}{\left\langle\sigma_{1}\right\rangle^{1 / 2}\left\langle\sigma_{2}\right\rangle^{1 / 2}\left\langle\xi_{2}\right\rangle^{k}}
$$

Case 2: $\left|\xi_{1}\right| \ll\left|\xi_{2}\right|$. This case is the same as Case 1 , since $J$ is symmetric with respect to $\xi_{1}$ and $\xi_{2}$.

Case 3: $\left|\xi_{1}\right| \sim\left|\xi_{2}\right|$. We have $\left|\xi_{3}\right| \lesssim\left|\xi_{1}\right|$, and the contribution of this case to $J$ is estimated by

$$
J_{3} \lesssim \int \frac{\left|\tilde{u}_{1} \tilde{u}_{2} \tilde{u}_{3}\right|\left\langle\xi_{3}\right\rangle^{3 / 2}}{\left\langle\sigma_{1}\right\rangle^{1 / 2}\left\langle\sigma_{2}\right\rangle^{1 / 2}\left\langle\sigma_{3}\right\rangle^{1 / 2}\left\langle\xi_{2}\right\rangle^{k}} \lesssim \int \frac{\left|\tilde{u}_{1} \tilde{u}_{2} \tilde{u}_{3}\right|}{\left\langle\sigma_{1}\right\rangle^{1 / 2}\left\langle\sigma_{2}\right\rangle^{1 / 2}\left\langle\xi_{2}\right\rangle^{k}}
$$

so this case again reduces to Case 1 .

Using the estimate (4.13) to $J_{1}$ and $J_{3}$, we thus obtain (4.26), and so (4.4).

Proof of (4.27). Set $b_{0}=\frac{1}{2}+\epsilon$ with small $\epsilon>0$ to be fixed later.

Case 1: $\left|\xi_{1}\right| \lesssim\left|\xi_{2}\right|$. In this case we have

$$
K_{1} \lesssim \int \frac{\left|\left\langle\sigma_{1}\right\rangle^{-1 / 2-\epsilon} \hat{w}_{1} \tilde{u}_{2} \tilde{u}_{3}\right|}{\left\langle\sigma_{1}\right\rangle^{1 / 2-\epsilon}\left\langle\sigma_{2}\right\rangle^{1 / 2}\left\langle\sigma_{3}\right\rangle^{1 / 2}\left\langle\xi_{3}\right\rangle^{k-3 / 2}} \lesssim \int \frac{\left|\left\langle\sigma_{1}\right\rangle^{-1 / 2-\epsilon} \hat{w}_{1} \tilde{u}_{2} \tilde{u}_{3}\right|}{\left\langle\sigma_{1}\right\rangle^{1 / 2-\epsilon}\left\langle\sigma_{2}\right\rangle^{1 / 2}\left\langle\xi_{3}\right\rangle^{k}} .
$$

Case 2: $\left|\xi_{3}\right| \sim\left|\xi_{1}\right| \gg\left|\xi_{2}\right|$. It is easy to see that

$$
K_{2} \lesssim \int \frac{\left|\left\langle\sigma_{1}\right\rangle^{-1 / 2-\epsilon} \hat{w}_{1} \tilde{u}_{2} \tilde{u}_{3}\right|}{\left\langle\sigma_{1}\right\rangle^{1 / 2-\epsilon}\left\langle\sigma_{2}\right\rangle^{1 / 2}\left\langle\xi_{2}\right\rangle^{k}}
$$

Notice that

$$
\left\|\left\langle\sigma_{1}\right\rangle^{-1 / 2-\epsilon} \hat{w}_{1}\right\|_{L^{2}} \lesssim\left\|w_{1}\right\|_{L^{2}}, \epsilon>0 .
$$

From the estimate (4.13) and (4.13') (let $\left.a_{1}=\frac{1}{2}-\epsilon, a_{2}=\frac{1}{2}, a_{3}=0\right)$, we know that if $k, \kappa$, and $\epsilon$ satisfy

$$
\begin{aligned}
& \frac{1}{2}+\epsilon<(1-\kappa)(1-\epsilon), \\
& k \geq \frac{5}{2}-\frac{(1-\kappa)(1-\epsilon)}{1 / 2+\epsilon} \geq 0,
\end{aligned}
$$

then $K_{1}+K_{2} \lesssim T^{\frac{\kappa}{2}(1-\epsilon)}\left\|w_{1}\right\|_{L^{2}}\left\|u_{2}\right\|_{L^{2}}\left\|u_{3}\right\|_{L^{2}}$. Since one can easily check that the condition (4.32) holds with $\epsilon$ and $\kappa$ selected sufficiently small provided that $k>\frac{1}{2}$, the estimate (4.27) (hence, the estimate (4.5)) follows immediately. 
To prove (4.28), one needs the following lemma.

Lemma 4.7. Assume that (4.29) holds and $\left|\xi_{1}\right| \gg\left|\xi_{2}\right| \gg 1$. Then there exists $c>0$ sufficiently small such that

$$
\left|\xi_{3}\right|^{3} \lesssim\left|\sigma_{1}\right|+\left|\sigma_{2}\right|+\left|\sigma_{3}\right| \chi_{c\left|\sigma_{3}\right| \leq\left|\xi_{3}\right|^{3} \leq\left|\sigma_{3}\right|} .
$$

Proof. From (4.29), we have

$$
\sigma_{1}-\sigma_{2} \mp \sigma_{3}=\left|\xi_{1}\right|^{2}-\left|\xi_{2}\right|^{2} \mp\left|\xi_{3}\right|^{3} .
$$

Since $\left|\xi_{1}\right| \gg\left|\xi_{2}\right| \gg 1$, one has $\left|\xi_{3}\right| \sim\left|\xi_{1}\right|$ and $\left|\xi_{3}\right|^{3} \lesssim\left|\sigma_{1}\right|+\left|\sigma_{2}\right|+\left|\sigma_{3}\right|$. Meanwhile, from the definition of $\sigma_{3}$, we have $\left|\xi_{3}\right|^{3} \leq\left|\sigma_{3}\right|$. We consider two cases.

Case 1: $\left|\xi_{3}\right|^{3}<c\left|\sigma_{3}\right|$. From (4.34), one gets

$$
\left|\sigma_{3}\right| \leq\left|\sigma_{1}\right|+\left|\sigma_{2}\right|+C\left|\xi_{3}\right|^{3} \leq\left|\sigma_{1}\right|+\left|\sigma_{2}\right|+C c\left|\sigma_{3}\right|,
$$

which yields $\left|\sigma_{3}\right| \lesssim\left|\sigma_{1}\right|+\left|\sigma_{2}\right|$ if $c>0$ is selected sufficiently small, such that $C c<1$. Then, we can obtain $\left|\xi_{3}\right|^{3} \lesssim\left|\sigma_{1}\right|+\left|\sigma_{2}\right|$.

Case 2: $\left|\sigma_{3}\right| \geq\left|\xi_{3}\right|^{3} \geq c\left|\sigma_{3}\right|$. Obviously

$$
\left|\xi_{3}\right|^{3} \lesssim\left|\sigma_{1}\right|+\left|\sigma_{2}\right|+\left|\sigma_{3}\right| \chi_{c\left|\sigma_{3}\right| \leq\left|\xi_{3}\right|^{3} \leq\left|\sigma_{3}\right|} .
$$

Combining Cases 1 and 2, we obtain the estimate (4.33) as desired.

Proof of (4.28). Set $b_{0}=\frac{1}{2}+\epsilon$ with $\epsilon>0$ small to be fixed later. We split the integral into two cases.

Case 1: $\left|\xi_{1}\right| \gg\left|\xi_{2}\right|$ or $\left|\xi_{1}\right| \ll\left|\xi_{2}\right|$. Since $L$ is symmetric with respect to $\xi_{1}$ and $\xi_{2}$, we only consider the case $\left|\xi_{1}\right| \gg\left|\xi_{2}\right|$. Moreover, we may assume $\left|\xi_{1}\right| \gg 1$, since the $\left|\xi_{1}\right| \lesssim 1$ case can be treated more easily. Using Lemma 4.7, the contribution of this case to $L$ can be estimated as

$$
\begin{aligned}
& L_{1} \lesssim \int \frac{\left|\tilde{u}_{1} \tilde{u}_{2} \hat{w}_{3}\right|\left\langle\xi_{3}\right\rangle^{3 / 2}}{\left\langle\sigma_{1}\right\rangle^{1 / 2}\left\langle\sigma_{2}\right\rangle^{1 / 2}\left\langle\sigma_{3}\right\rangle\left\langle\xi_{2}\right\rangle^{k}} \\
& \lesssim \int \frac{\left|\tilde{u}_{1} \tilde{u}_{2} \hat{w}_{3}\right|\left(\left\langle\sigma_{1}\right\rangle^{1 / 2}+\left\langle\sigma_{2}\right\rangle^{1 / 2}+\left\langle\sigma_{3}\right\rangle^{1 / 2} \chi_{c\left|\sigma_{3}\right| \leq\left|\xi_{3}\right|^{3} \leq\left|\sigma_{3}\right|}\right)}{\left\langle\sigma_{1}\right\rangle^{1 / 2}\left\langle\sigma_{2}\right\rangle^{1 / 2}\left\langle\sigma_{3}\right\rangle\left\langle\xi_{2}\right\rangle^{k}} \\
& \lesssim \int \frac{\left|\tilde{u}_{1} \tilde{u}_{2}\left\langle\sigma_{3}\right\rangle^{-1 / 2-\epsilon} \hat{w}_{3}\right|}{\left\langle\sigma_{2}\right\rangle^{1 / 2}\left\langle\sigma_{3}\right\rangle^{1 / 2-\epsilon}\left\langle\xi_{2}\right\rangle^{k}}+\int \frac{\left|\tilde{u}_{1} \tilde{u}_{2}\left\langle\sigma_{3}\right\rangle^{-1 / 2-\epsilon} \hat{w}_{3}\right|}{\left\langle\sigma_{1}\right\rangle^{1 / 2}\left\langle\sigma_{3}\right\rangle^{1 / 2-\epsilon}\left\langle\xi_{2}\right\rangle^{k}} \\
& +\int \frac{\mid \tilde{u}_{1} \tilde{u}_{2}\left\langle\sigma_{3}\right\rangle^{-1 / 2} \chi_{c\left|\sigma_{3}\right| \leq\left|\xi_{3}\right|^{3} \leq\left|\sigma_{3}\right| \hat{w}_{3} \mid}}{\left\langle\sigma_{1}\right\rangle^{1 / 2}\left\langle\sigma_{2}\right\rangle^{1 / 2}\left\langle\xi_{2}\right\rangle^{k}} \\
& =: L_{11}+L_{12}+L_{13} \text {. }
\end{aligned}
$$

Case 2: $\left|\xi_{1}\right| \sim\left|\xi_{2}\right|$. Then the contribution of this case to $L$ is estimated by

$$
L_{2} \lesssim \int \frac{\left|\tilde{u}_{1} \tilde{u}_{2} \hat{w}_{3}\right|\left\langle\xi_{3}\right\rangle^{3 / 2}}{\left\langle\sigma_{1}\right\rangle^{1 / 2}\left\langle\sigma_{2}\right\rangle^{1 / 2}\left\langle\sigma_{3}\right\rangle^{1 / 2}\left\langle\xi_{2}\right\rangle^{k}},
$$

so it is easy to see that this case reduces to Case 1.

Note that $\left\|\left\langle\sigma_{3}\right\rangle^{-1 / 2} \chi_{c\left|\sigma_{3}\right| \leq\left|\xi_{3}\right|^{3} \leq\left|\sigma_{3}\right|} \hat{w}_{3}\right\|_{L^{2}} \lesssim\left\|w_{3}\right\|_{L^{2}}$. Now applying Lemma 4.6 to the terms $L_{11}, L_{12}$, and $L_{13}$, one can easily see that if $k>\frac{1}{2}$, then the estimate (4.28) holds for some $\theta_{2}>0$. 
Finally, for the integral equations (3.1)-(3.3), we use the linear estimates (3.5), (3.6), (3.8), (3.11), (3.12), and the bilinear estimates given in Propositions 4.1-4.2, and a standard contraction argument, to deduce that the system (2.2) has a unique local solution $(\mathcal{E}, N, \mathcal{B}) \in X_{S}^{k, \frac{1}{2}}(T) \times X_{W}^{l, \frac{1}{2}}(T) \times X_{H}^{k-\frac{3}{2}, \frac{1}{2}}(T)$. Moreover, from (3.7) and (3.13), we have $(\mathcal{E}, N, \mathcal{B}) \in C\left([0, T] ; H^{k} \times H^{l} \times H^{k-\frac{3}{2}}\right)$. Hence, this completes the proof of Theorem 1.1 .

Acknowledgment. L. Han is supported by the National Science Foundation of China (Grant 11001022, 11071240), the Spectical Funds for Co-construction Project of Beijing, and the Fundamental Research Funds for the Central Universities. J. Zhang is supported by Zhejiang Provincial Natural Science Foundation (Grant No. LQ12A01013) and the National Science Foundation of China (Grant 11201185). Z. Gan is supported by the National Science Foundation of China (Grant 11171241).

\section{Appendix A. Proof of Lemma 3.1 and Lemma 3.2.}

Proof of Lemma 3.1. For $0 \leq b \leq 1$,

$$
\begin{aligned}
&\left\|\langle|\tau|+\varphi(\xi)\rangle^{b} \mathscr{F}_{t}\left(\psi(t) e^{-|t| \varphi(\xi)}\right)\right\|_{L_{\tau}^{2}} \\
& \lesssim\left\|\langle\tau\rangle^{b} \mathscr{F}_{t}\left(\psi(t) e^{-|t| \varphi(\xi)}\right)\right\|_{L_{\tau}^{2}}+\langle\varphi(\xi)\rangle^{b}\left\|\mathscr{F}_{t}\left(\psi(t) e^{-|t| \varphi(\xi)}\right)\right\|_{L_{\tau}^{2}}
\end{aligned}
$$

From scale invariance, we have $\|f(\lambda t)\|_{\dot{H}^{b}(\mathbb{R})} \sim \lambda^{b-1 / 2}\|f(t)\|_{\dot{H}^{b}(\mathbb{R})}, b \geq 0$. When $|\xi| \geq 1$, using the fact

$$
\langle\tau\rangle^{b} \leq C\left(\left\langle\tau-\tau_{1}\right\rangle^{b}+\left|\tau_{1}\right|^{b}\right)
$$

and Young's inequality, we have

$$
\begin{aligned}
&\left\|\langle\tau\rangle^{b} \mathscr{F}_{t}\left(\psi(t) e^{-|t| \varphi(\xi)}\right)\right\|_{L_{\tau}^{2}} \\
& \lesssim\left\|\langle\tau\rangle^{b} \mathscr{F}_{t} \psi\right\|_{L^{1}}\left\|e^{-\varphi(\xi)|t|}\right\|_{L^{2}}+\left\|\mathscr{F}_{t} \psi\right\|_{L^{1}}\left\|e^{-\varphi(\xi)|t|}\right\|_{\dot{H}^{b}} \lesssim|\varphi(\xi)|^{b-\frac{1}{2}}
\end{aligned}
$$

When $|\xi| \leq 1$, noticing that $\left\||t|^{n} \psi(t)\right\|_{H^{b}} \leq\left\||t|^{n} \psi(t)\right\|_{H^{1}} \leq C 2^{n}, n \geq 1$, we have

$$
\left\|\langle\tau\rangle^{b} \mathscr{F}_{t}\left(\psi(t) e^{-|t| \varphi(\xi)}\right)\right\|_{L_{\tau}^{2}} \leq\left\|\psi(t) e^{-|t| \varphi(\xi)}\right\|_{H^{b}} \leq \sum_{n=0}^{\infty} \frac{|\varphi(\xi)|^{n}}{n !}\left\|||^{n} \psi(t)\right\|_{H^{b}} \leq C .
$$

Taking $b=1 / 2$ or $b=0$, and combining with (A.1), we have

$$
\left\|\langle|\tau|+\varphi(\xi)\rangle^{1 / 2} \mathscr{F}_{t}\left(\psi(t) e^{-|t| \varphi(\xi)}\right)\right\|_{L_{\tau}^{2}} \leq C .
$$

Proof of Lemma 3.2. For the function $g(\xi, t)$, we consider four different cases:

$$
\begin{aligned}
g(\xi, t)= & \psi(t) \int_{|\tau| \leq 1} \frac{e^{\mathrm{i} t \tau}-1}{\mathrm{i} \tau+\varphi(\xi)} \hat{f}(\tau, \xi) d \tau+\psi(t) \int_{|\tau| \leq 1} \frac{1-e^{-\varphi(\xi)|t|}}{\mathrm{i} \tau+\varphi(\xi)} \hat{f}(\tau, \xi) d \tau \\
& \quad+\psi(t) \int_{|\tau| \geq 1} \frac{e^{\mathrm{i} t \tau}}{\mathrm{i} \tau+\varphi(\xi)} \hat{f}(\tau, \xi) d \tau-\psi(t) \int_{|\tau| \geq 1} \frac{e^{-\varphi(\xi)|t|}}{\mathrm{i} \tau+\varphi(\xi)} \hat{f}(\tau, \xi) d \tau \\
= & : I_{1}+I_{2}+I_{3}-I_{4} .
\end{aligned}
$$


We will estimate terms $I_{i}, i=1, \ldots, 4$, respectively. First, by (A.2),

$$
\begin{aligned}
&\left\|\langle\mathrm{i} \tau+\varphi(\xi)\rangle^{1 / 2} \mathscr{F}_{t}\left(I_{3}\right)\right\|_{L_{\tau}^{2}}^{2}=\int_{\mathbb{R}}\langle\mathrm{i} \tau+\varphi(\xi)\rangle\left|\hat{\psi}\left(\tau^{\prime}\right) *\left(\frac{\hat{f}\left(\tau^{\prime}, \xi\right)}{\mathrm{i} \tau^{\prime}+\varphi(\xi)} \chi_{\left|\tau^{\prime}\right| \geq 1}\right)(\tau)\right|^{2} d \tau \\
& \leq\left\|\left|\left\langle\tau^{\prime}\right\rangle^{1 / 2} \hat{\psi}\left(\tau^{\prime}\right)\right| *\left(\frac{\left|\hat{f}\left(\tau^{\prime}, \xi\right)\right|}{\left|\mathrm{i} \tau^{\prime}+\varphi(\xi)\right|} \chi_{\left|\tau^{\prime}\right| \geq 1}\right)\right\|_{L^{2}}^{2} \\
&+\left\|\left|\hat{\psi}\left(\tau^{\prime}\right)\right| *\left(\frac{\left|\hat{f}\left(\tau^{\prime}, \xi\right)\right|}{\left.\left|\mathrm{i} \tau^{\prime}+\varphi(\xi)\right|\right|^{1 / 2}} \chi_{\left|\tau^{\prime}\right| \geq 1}\right)\right\|_{L^{2}}^{2} \\
& \leq C\left\|\frac{\hat{f}\left(\tau^{\prime}, \xi\right)}{\left\langle\mathrm{i} \tau^{\prime}+\varphi(\xi)\right\rangle^{1 / 2}}\right\|_{L^{2}}^{2} .
\end{aligned}
$$

Then by Lemma 3.2, we have

$$
\begin{aligned}
& \left\|\langle\mathrm{i} \tau+\varphi(\xi)\rangle^{1 / 2} \mathscr{F}_{t}\left(I_{4}\right)\right\|_{L_{\tau}^{2}}^{2} \\
\leq & \left(\int_{\mathbb{R}} \frac{|\hat{f}(\tau, \xi)|}{\langle\mathrm{i} \tau+\varphi(\xi)\rangle} d \tau\right)^{2} \int_{\mathbb{R}}\langle\mathrm{i} \tau+\varphi(\xi)\rangle\left|\mathscr{F}_{t}\left(\psi(t) e^{-\varphi(\xi)|t|}\right)\right|^{2}(\tau) d \tau \\
\leq & C\left(\int_{\mathbb{R}} \frac{|\hat{f}(\tau, \xi)|}{\langle\mathrm{i} \tau+\varphi(\xi)\rangle} d \tau\right)^{2} .
\end{aligned}
$$

For the term $I_{2}$, note that

$$
\begin{aligned}
& \left\|\langle\mathrm{i} \tau+\varphi(\xi)\rangle^{1 / 2} \mathscr{F}_{t}\left(I_{2}\right)\right\|_{L_{\tau}^{2}}^{2} \\
\leq & \left(\int_{\mathbb{R}} \frac{|\hat{f}(\tau, \xi)|^{2}}{\langle\mathrm{i} \tau+\varphi(\xi)\rangle} d \tau\right)\left(\int_{|\tau| \leq 1} \frac{\langle\mathrm{i} \tau+\varphi(\xi)\rangle}{|\mathrm{i} \tau+\varphi(\xi)|^{2}} d \tau\right)\left\|\langle\mathrm{i} \tau+\varphi(\xi)\rangle^{1 / 2} \mathscr{F}_{t}\left(\psi(t)\left(1-e^{-\varphi(\xi)|t|}\right)\right)(\tau)\right\|_{L_{\tau}^{2}}^{2}
\end{aligned}
$$

When $|\varphi(\xi)| \geq 1$, Lemma 3.2 implies that

$$
\begin{aligned}
\left\|\langle\mathrm{i} \tau+\varphi(\xi)\rangle^{1 / 2} \mathscr{F}_{t}\left(\psi(t)\left(1-e^{-\varphi(\xi)|t|}\right)\right)(\tau)\right\|_{L_{\tau}^{2}}^{2} & \leq\left\|\langle\mathrm{i} \tau+\varphi(\xi)\rangle^{1 / 2} \mathscr{F}_{t} \psi(t)\right\|_{L_{\tau}^{2}}^{2}+C \\
& \leq C\langle\varphi(\xi)\rangle .
\end{aligned}
$$

When $|\varphi(\xi)| \leq 1$, using Taylor's expansion and $\left\||t|^{n} \psi(t)\right\|_{H^{1 / 2}} \leq\left\||t|^{n} \psi(t)\right\|_{H^{1}} \leq C 2^{n}$, we have

$$
\left\|\langle\mathrm{i} \tau+\varphi(\xi)\rangle^{1 / 2} \mathscr{F}_{t}\left(\psi(t)\left(1-e^{-\varphi(\xi)|t|}\right)\right)(\tau)\right\|_{L_{\tau}^{2}}^{2} \leq\left\|\psi(t)\left(1-e^{-\varphi(\xi)|t|}\right)\right\|_{H_{t}^{1 / 2}}^{2} \leq C|\varphi(\xi)|^{2}
$$

Moreover, one can easily see that

$$
\int_{|\tau| \leq 1} \frac{\langle\mathrm{i} \tau+\varphi(\xi)\rangle}{|\mathrm{i} \tau+\varphi(\xi)|^{2}} d \tau \lesssim \begin{cases}|\varphi(\xi)|^{-1}, & \text { if } \varphi(\xi) \geq 1 \\ |\varphi(\xi)|^{-2}, & \text { if } \varphi(\xi) \leq 1\end{cases}
$$

Putting the above three estimates into (A.5), we get

$$
\left\|\langle\mathrm{i} \tau+\varphi(\xi)\rangle^{1 / 2} \mathscr{F}_{t}\left(I_{2}\right)\right\|_{L_{\tau}^{2}}^{2} \leq C \int_{\mathbb{R}} \frac{|\hat{f}(\tau, \xi)|^{2}}{\langle\mathrm{i} \tau+\varphi(\xi)\rangle} d \tau .
$$


Using Taylor's expansion to $e^{\mathrm{i} t \tau}-1$ again, as in the argument in $I_{2}$ we have

$$
\begin{aligned}
\left\|\langle\mathrm{i} \tau+\varphi(\xi)\rangle^{1 / 2} \mathscr{F}_{t}\left(I_{1}\right)\right\|_{L_{\tau}^{2}}^{2} & \leq\left(\sum_{n \geq 1}(1+\varphi(\xi))^{1 / 2}\left\|\frac{t^{n} \psi(t)}{n !}\right\|_{H_{t}^{1 / 2}} \int_{|\tau| \leq 1} \frac{|\mathrm{i} \tau|^{n}|\hat{f}(\tau, \xi)|}{|\mathrm{i} \tau+\varphi(\xi)|} d \tau\right)^{2} \\
& \leq C\langle\varphi(\xi)\rangle \int_{|\tau| \leq 1} \frac{|\tau|^{2}\langle\mathrm{i} \tau+\varphi(\xi)\rangle}{|\mathrm{i} \tau+\varphi(\xi)|^{2}} d \tau \int_{\mathbb{R}} \frac{|\hat{f}(\tau, \xi)|^{2}}{\langle\mathrm{i} \tau+\varphi(\xi)\rangle} d \tau \\
& \leq C \int_{\mathbb{R}} \frac{|\hat{f}(\tau, \xi)|^{2}}{\langle\mathrm{i} \tau+\varphi(\xi)\rangle} d \tau
\end{aligned}
$$

Hence, Lemma 3.3 follows from (A.3), (A.4), (A.6), and (A.7).

\section{REFERENCES}

[1] H. Added and S. Added, Existence globle de solutions fortes pour les équations de la turbulence de Langmuir en dimension 2, C.R. Acad. Sci. Paris, 299, 551-554, 1984.

[2] H. Added and S. Added, Equations of Langmuir turbulence and nonlinear Schrödinger equation: Smoothness and approximation, J. Funct. Anal., 1, 183-210, 1998.

[3] J. Bourgain, Fourier transform restriction phenomena for certain lattice subsets and application to nonlinear evolution equations I. Schrödinger, Geom. Funct. Anal., 3, 107-156, 1993.

[4] J. Bourgain, Fourier transform restriction phenomena for certain lattice subsets and application to nonlinear evolution equations II. The KdV equation, Geom. Funct. Anal., 3, 209-262, 1993.

[5] J. Bourgain and J. Colliander, On wellposedness of the Zakharov system, Internat. Math. Res. Notices, 11, 515-546, 1996.

[6] I. Bejenaru, S. Herr, J. Holmer, and D. Tataru, On the $2 d$ Zakharov system with $L^{2}$ Schrödinger data, Nonlin., 22, 1063-1089, 2009.

[7] I. Bejenaru and S. Herr, Convolutions of singular measures and applications to the Zakharov system, J. Funct. Anal., 261, 478-506, 2011.

[8] J. Colliander, M. Keel, G. Staffilani, H. Takaoka, and T. Tao, Sharp global well-posedness for $K d V$ and modified $K d V$ on $\mathbb{R}$ and $\mathbb{T}$, J. Amer. Math. Soc., 3, 705-749, 2003.

[9] B. Guo and L. Shen, The existence and uniqueness of the classical solution on the periodic initial value problem for Zakharov equation, (in Chinese), Acta Mathematicae Applicatae Sinica, 3, 310-324, 1982.

[10] L. Han, J. Zhang, Z. Gan, and B. Guo, On the limit behavior of the magnetic Zakharov system, Sci. China Math., 55, 509-540, 2012.

[11] B. Guo, L. Han, and Z. Gan, Cauchy problem for the Zakharov system arising from ion-acoustic modes with low regularity data, J. Appl. Anal. Comut., 2, 11-28, 2012.

[12] B. Guo and J. Zhang, Well-posedness of the Cauchy problem for the magnetic Zakharov type system, Nonlin., 24, 2191-2210, 2011.

[13] X.T. He, The pondermotive force and magnetic field generation effects resulting from the nonlinear interaction between plasma-wave and particles (in Chinese), Acta Phys. Sinica, 32, 325-337, 1983.

[14] J. Ginibre, Y. Tsutsumi, and G. Velo, On the Cauchy problem for the Zakharov system, J. Funct. Anal., 2, 384-436, 1997.

[15] M. Kono, M.M. Skoric, and D. Ter Haar, Spontaneous excitation of magnetic fields and collapse dynamics in a Langmuir plasma, J. Plasma Phys., 26, 123-146, 1981.

[16] C. Kenig and W. Wang, Existence of local smooth solution for a generalized Zakharov system, J. Fourier Anal. Appl., 4, 469-490, 1998.

[17] C. Laurey, The Cauchy problem for a generalized Zakharov system, Diff. Int. Equ., 1, 105-130, 1995.

[18] L. Glangetas and F. Merle, Existence of self-similar blow-up solutions for Zakharov equation in dimension two. Part I, Comm. Math. Phys., 160, 173-215, 1994.

[19] L. Glangetas and F. Merle, Concentration properties of blow-up solutions and instability results for Zakharov equation in dimension two. Part II, Comm. Math. Phys., 160, 349-389, 1994.

[20] N. Masmoudi and K. Nakanishi, From the Klein-Gordon-Zakharov system to the nonlinear Schrödinger equation, J. Hyperbolic Diff. Equ., 4, 975-1008, 2005. 
[21] N. Masmoudi and K. Nakanishi, Energy convergence for singular limits of Zakharov type systems, Invent. Math., 172, 535-583, 2008.

[22] L. Molinet and F. Ribaud, On the low regularity of the Korteweg-de Vries-Burgers equation, Int. Math. Res. Not., 37, 1979-2005, 2002.

[23] H. Pecher, Global solutions with infinite energy for the one-dimensional Zakharov system, Electron. J. Differ. Eqs., 41, 1-18, 2005.

[24] C. Sulem and P.L. Sulem, Quelques résulatats de régularité pour les équation de la turbulence de Langmuir, C.R. Acad. Sci. Paris, 289, 173-176, 1979.

[25] S.H. Schochet and M.I. Weinstein, The nonlinear Schrödinger limit of the Zakharov equations governing Langmuir turbulence, Commun. Math. Phys., 106, 569-580, 1986.

[26] V.E. Zakharov, Collapse of Langmuir waves, Sov. Phys. JETP., 35, 908-914, 1972. 